\title{
Cytogenetic characterization of the strongly electric Amazonian eel, Electrophorus electricus (Teleostei, Gymnotiformes), from the Brazilian rivers Amazon and Araguaia
}

\author{
Soraia B.A. Fonteles ${ }^{1}$, Carlos E. Lopes ${ }^{1}$, Alberto Akama ${ }^{2}$, Flora M.C. Fernandes ${ }^{1}$, \\ Fábio Porto-Foresti ${ }^{3}$, José A. Senhorini ${ }^{4}$, Maria de Fátima Z. Daniel-Silva ${ }^{1}$, \\ Fausto Foresti ${ }^{5}$ and Lurdes Foresti de Almeida-Toledo ${ }^{1}$ \\ ${ }^{1}$ Departamento de Genética e Biologia Evolutiva, Instituto de Biociências, Universidade de São Paulo, \\ São Paulo, SP, Brazil. \\ ${ }^{2}$ Museu de Zoologia, Universidade de São Paulo, São Paulo, SP, Brazil. \\ ${ }^{3}$ Departamento de Ciências Biológicas, Faculdade de Ciências de Bauru, \\ Universidade Estadual Paulista, Bauru, SP, Brazil. \\ ${ }^{4}$ Centro de Pesquisa e Gestão de Recursos Pesqueiros Continentais, \\ Instituto Brasileiro do Meio Ambiente e dos Recursos Naturais Renováveis, Pirassununga, SP, Brazil. \\ ${ }^{5}$ Departamento de Morfologia, Instituto de Biociências, Universidade Estadual Paulista, Botucatu, \\ SP, Brazil.
}

\begin{abstract}
A karyotype analysis of the electric eel, Electrophorus electricus (Teleostei, Gymnotiformes), a strongly electric fish from northern South America, is presented. Two female specimens were analyzed, one from the Amazon River and one from the Araguaia River. The specimens had a chromosomal number of $2 n=52(42 M-S M+10 A)$. C-bands were present in a centromeric and pericentromeric position on part of the chromosomes; some interstitial C-bands were also present. Heteromorphic nucleolus organizer regions (NORs) were detected in two chromosome pairs of the specimen from the Amazon River. The chromosome number and karyotype characteristics are similar to those of other Gymnotidae species. The genera Electrophorus and Gymnotus are positioned as the basal lineages in the Gymnotiformes phylogeny.
\end{abstract}

Key words: chromosome, fish, Gymnotidae, electric eel.

Received: September 26, 2006; Accepted: April 18, 2007.

Electrophorus electricus Gill, 1864, is the only strongly electric Neotropical species of Gymnotiformes. This species is commonly known as the electric eel or "poraquê". Larger adults of this species are able to produce shocks approaching 500-600 V (Campos da Paz, 2003). E. electricus is found in the northern areas of South America, including the Guyanas and the Orinoco River, as well as the middle and lower Amazon basin (Campos da Paz, 2003). The body of E. electricus is long and snake-like, without scales, lacking caudal, dorsal and pelvic fins. Adult individuals can grow as long as 2.5 meters. They are obligatory air breathers. The strong electric organ discharges are usually used for predation and defensive purposes. Weak electric

Send correspondence to Lurdes Foresti de Almeida Toledo. Departamento de Genética e Biologia Evolutiva, Instituto de Biociências, Universidade de São Paulo, Caixa Postal 11.461, 05422-970 São Paulo, SP, Brazil. E-mail: Iftoledo@ib.usp.br. organ discharges are used for electrolocation and identification of foreign objects (Moller, 1995; Val and Almeida-Val, 1995; Berra, 2001).

E. electricus can be very dangerous to humans because of its strong electric capabilities, and this species has little economic value. Even though, E. electricus has been a constant object of study for many years (Moller, 1995). This species is mainly investigated concerning its taxonomy (Alves-Gomes et al., 1995; Albert and Campos da Paz, 1998; Albert, 2001), behavior (Moller, 1995; Berra, 2001), and electric organ discharges (Brown, 1957; Keynes, 1957; Moller, 1995; Berra, 2001). E. electricus was recently included in the family Gymnotidae (Albert, 2001) and, according to this classification, Gymnotidae is composed by two genera, Electrophorus and Gymnotus.

Cytogenetic studies have been carried out in Gymnotiformes, focusing mainly the families 
Sternopygidae and Gymnotidae. A wide variability in chromosome number was found to occur among species and populations of this fish group (Almeida-Toledo et al., 2007). Cytogenetic information on E. electricus is, however, very scarce in the literature. In the present work, we describe the karyotype of E. electricus and discuss its possible relationship with other Gymnotidae species.

Electrophorus electricus is a species extremely difficult to handle because of its strong electric discharges (Campos da Paz, 2003). This fact explains the difficulty of getting chromosome preparations from these animals. For our study, two female specimens of E. electricus were collected, one from the Amazon River (Almeirim, Pará State), and the other one from the Araguaia River (Nova Crixás, Goiás State). The specimen from the Amazon River was identified by Dr. Alberto Akama (Instituto Nacional de Pesquisas da Amazônia, Manaus, AM, Brazil) and was deposited at the Museum of Zoology of the University of São Paulo, Brazil (MZUSP 84570). The specimen from the Araguaia River was deposited at the Collection of Fish Biology and Genetics of the Department of Morphology, São Paulo State University (Botucatu, SP, Brazil).

Mitotic chromosome preparations were obtained according to Foresti et al. (1993): kidney cells were suspended in $7 \mathrm{~mL}$ Hank's solution with two drops of $0.02 \%$ colchicine for $15 \mathrm{~min}$, then centrifuged; the pellet was resuspended in $0.075 \mathrm{M} \mathrm{KCl}$ and incubated at $37{ }^{\circ} \mathrm{C}$ for $30 \mathrm{~min}$. The cell suspension was then centrifuged and the pellet was resuspended in fixative solution (methanol:acetic acid 3:1) and centrifuged twice. The final pellet was resuspended in fresh fixative and dropped onto warmed slides. The slides were analyzed after conventional Giemsa staining, C-banding (Sumner, 1972), and silver staining of nucleolus organizer regions (Ag-NOR) (Howell and Black, 1980).

Conventional chromosome staining - The analyzed female specimen of E. electricus from the Amazon River had a basic karyotype of 52 chromosomes. These chromosomes could be arranged into 26 pairs (Figure 1), forming three groups: a group of 14 pairs of larger metacentric-submetacentric chromosomes, including two heteromorphic pairs formed by two submetacentric chromosomes of different sizes (pairs 2 and 4), a group of small metacentric elements of about the same size (pairs 15 to 21), and a group of five subtelocentric-acrocentric chromosomes. Pairs number 1-15 presented a slight decrease in chromosome length from the submetacentric pair number one to the small metacentric pair number 15 . The heteromorphic pair number 2 is composed by NOR bearing chromosomes, and one of the homologues presented a duplication of this region. As shown in Figure 1, the NOR region is flanked by two C-band positive blocks, but the NOR is C-band negative. The secondary constriction was easily identified in the two homologues of pair 2. The chromosomes of pair number 4 are heteromorphic due to the presence of a hetero-
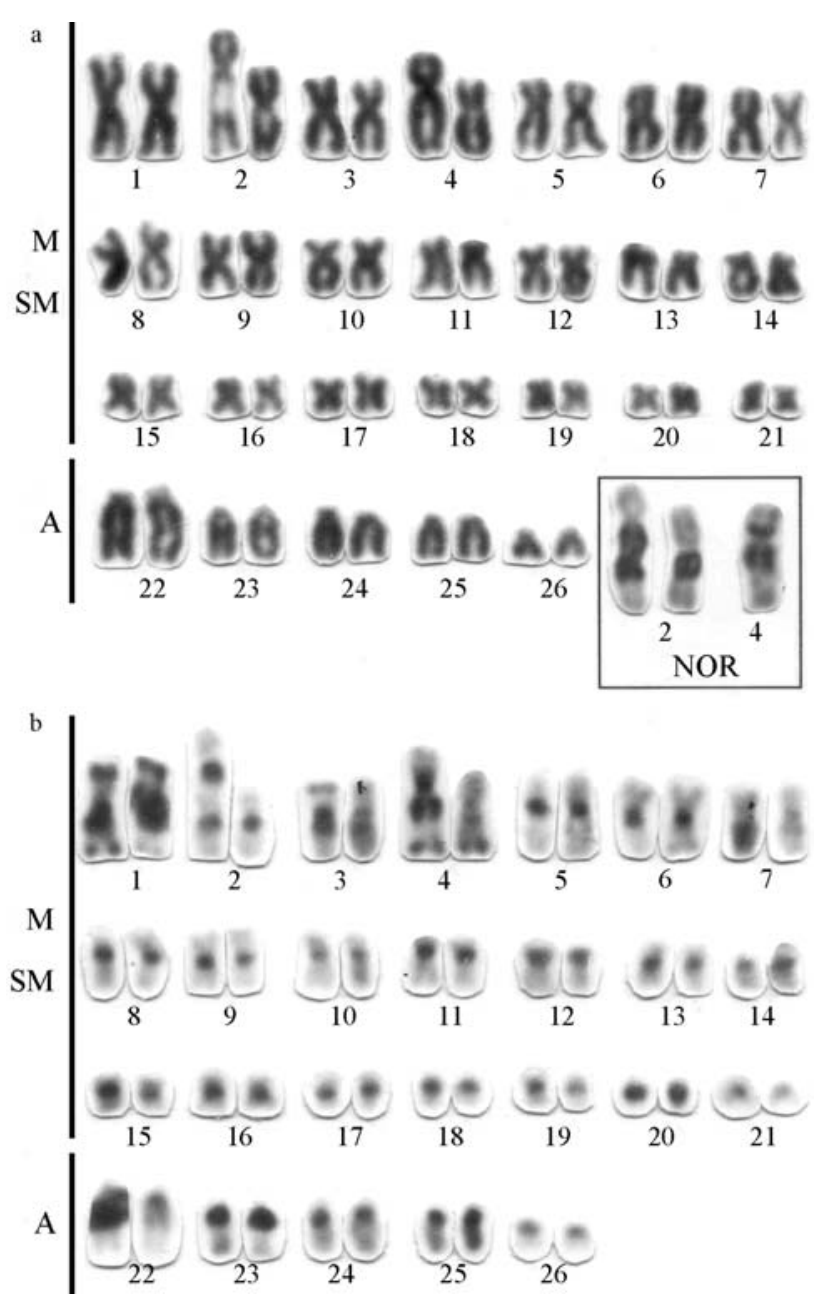

Figure 1 - Karyotype of Electrophorus electricus from the Amazon River. a) Giemsa stained chromosomes; b) C-banded karyotype. In the insert, silver-stained NORs: pair 2 evidencing strongly heteromorphic NOR bearing chromosomes; pair 4 presents the NOR in only one of the homologues.

chromatic NOR in one of the homologues, which is absent in the other homologue. In this case, the NOR is C-band positive and is present both in the proximal region of the short arm and of the long arm of one of the chromosomes of this pair; the other member of the pair 4 has just a small heterochromatic mark in this region. The specimen from the Araguaia River had the same chromosome number presenting a group of metacentric-submetacentric chromosomes (pairs 1 to 15), a group of small metacentric chromosomes (pairs 16-21), and a group of five subtelocentricacrocentric chromosome pairs (pairs 22-26) (Figure 2). Chromosome pair numbers 2 and 4 did not show any heteromorphism.

C-band analysis - The C-banded karyotype of $E$. electricus from the Amazon River exhibited a great number of constitutive heterochromatic regions (Figure 1). Constitutive heterochromatin was located in the pericentromeric region of all chromosomes. Pair 1 presented heterochromatic blocks at telomeric regions of both short and long arms, and a large heterochromatic block in the proximal re- 
gion of the long arm. The heteromorphic pair 2, one of the NOR-bearing pairs, had heterochromatic blocks associated to NORs, but the NOR was C-band-negative. Larger blocks were present at the proximal portion of both arms of one of the homologues and at the telomeric region of the long arm in both homologues of pair 4 (Figure 1). The specimen from the Araguaia River presented heterochromatic blocks located in the pericentromeric regions of most of the biarmed chromosomes (Figure 2).

Nucleolus organizer regions (NORs) - Specific staining of NORs with $\mathrm{AgNO}_{3}$ (Figure 1 - insert) of the specimen from the Amazon River revealed a large NOR in the proximal region of the long arm of one of the homologues of pair 2, flanked by two heterochromatic blocks; the other homologue presented a smaller NOR. The silver staining also indicated the presence of a NOR at the pericentromeric region of one of the homologues of pair 4, including the proximal region of both arms. The specimen from the Araguaia River had NORs present in the proximal region of the long arm in both homologues of pair 1 (Figure 2).

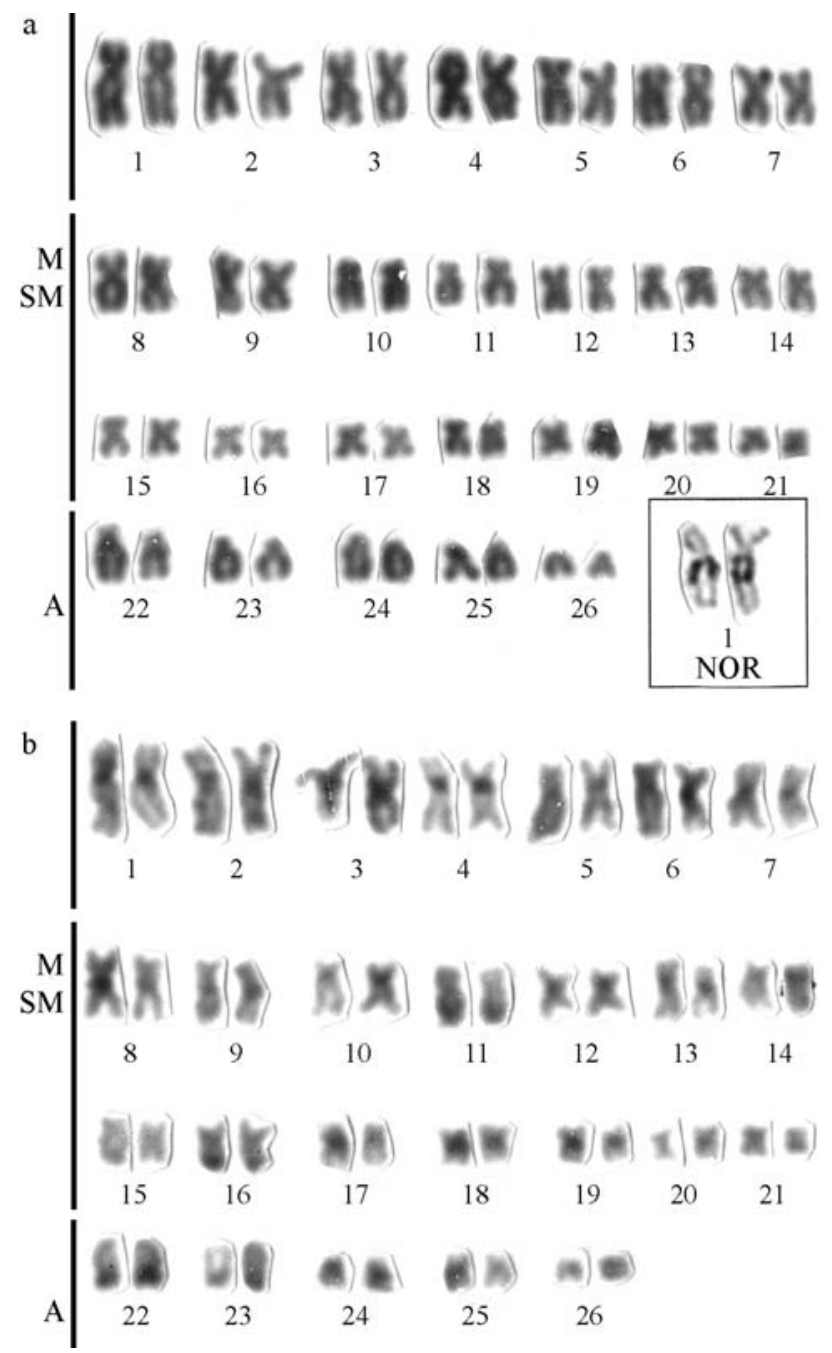

Figure 2 - Karyotype of Electrophorus electricus from the Araguaia River. a) Giemsa stained chromosomes; b) C-banded karyotype. In the insert, silver-stained NORs.
In contrast to numerous reports on taxonomy, behavior, and electric organ discharge, cytogenetic studies of $E$. electricus are still incipient. Preliminary results on this species have been reported by Medaglia et al. (1995, personal communication), disclosing a karyotype with $2 \mathrm{n}=52$ chromosomes. Our present analysis in specimens from two different river basins confirms this chromosome number.

The chromosomal numbers already described in Gymnotiformes vary from $2 \mathrm{n}=24$ for Apteronotus albifrons (Almeida-Toledo et al., 1981); Almeida-Toledo et al., 1981) to $2 \mathrm{n}=54$, found in Gymnotus carapo (Foresti et al., 1984). In Gymnotidae, chromosome numbers range from $2 \mathrm{n}=40$, present in Gymnotus sylvius, to $2 \mathrm{n}=54$, in $G$. carapo (Fernandes-Matioli et al., 1998). The karyotypes already described for Gymnotiformes are all speciesspecific, and all the species analyzed so far presented only one pair of nucleolous organizer regions. The constitutive heterochromatin distribution has different patterns in families Sternopygidae and Gymnotidae: while the amount of heterochromatin in the former is relatively small (Almeida-Toledo et al., 2002), the latter is characterized, in general, by the occurrence of large blocks of heterochromatin dispersed over all chromosomes (Fernandes-Matioli and Almeida-Toledo, 2001).

The three active NORs found in the present analysis in the specimen of E. electricus from the Amazon River may be a sporadic occurrence, since the specimen from the Araguaia River, and the two specimens already analyzed by Medaglia et al. (1995, personal communication) had only one pair of NORs. The presence of one pair of NORs is also a character of all the other Gymnotiformes analyzed to date. On the other hand, the occurrence of heterochromatic blocks flanking the NORs as found in the specimen from the Amazon River is not a common feature in Gymnotidae. In addition, chromosome pair 4 presents a C-positive NOR region in one of the homologues, with no correspondence in the other homologue.

The sample of Amazon River and the sample of Araguaia River show the same chromosome composition, with 21 pairs of metacentric-submetacentric chromosomes and five pairs of subtelocentric-acrocentric chromosomes. The Amazon and the Araguaia karyotypes differ in relation to the $\mathrm{C}$ band pattern: while large $\mathrm{C}$-band blocks are found in all the chromosome pairs in the Amazonian specimen, in the Araguaia specimen they are present as small pericentromeric blocks in most of the metacentric and submetacentric chromosomes, and in the distal region of the long arm of chromosomes $11,16,22$, and 23 , but are hardly noticed in the other subtelocentric-acrocentric chromosomes. In addition, the heterochromatin pattern of the Amazonian specimen allowed the identification of large heterochromatic blocks associated with pairs 2 and 4 .

In relation to the nucleolus organizer region, another good chromosome marker, the Araguaia specimen presents a very clear Ag-positive signal in the proximal region of the 
long arm of the large submetacentric pair 1, while the Amazonian specimen has three NOR-bearing chromosomes. These three NORs are very different in size and, together with the heterochromatic blocks already mentioned, result in the heteromorphism detected after Giemsa staining and C-banding in chromosome pairs 2 and 4, as shown in Figures 1 and 2. Taken together, the heterochromatic blocks flanking the NOR region, the NOR size difference between the two homologues of the chromosome pair 2, and the occurrence of an heterochromatic NOR in only one of the homologues of pair 4 of the Amazonian specimen are responsible for the differences between the homologues of pairs 2 and 4 .

It is possible to conclude that, although the karyotypes of the Amazon and of the Araguaia specimens are similar, they present some differences related to NOR size and the number of these cistrons, as well as to the heterochromatin distribution in two chromosome pairs.

The Gymnotidae genera Electrophorus and Gymnotus are positioned as the basal lineages of the Gymnotiformes phylogeny (e.g., Alves-Gomes et al., 1995; Albert, 2001). The comparison of the karyotype of $E$. electricus with those of $G$. carapo $(2 \mathrm{n}=54)$ (Foresti et al.,1984), and those of $G$. pantherinus $(2 \mathrm{n}=52), G$. inaequilabiatus $(2 \mathrm{n}=52)$ and $G$. sylvius $(2 \mathrm{n}=40)$ (Fernandes-Matioli et al., 1998) reveals a proximity in chromosome number within the first three species. However, while only metacentric and submetacentric chromosomes are found in the karyotypes of the other Gymnotidae, E. electricus presents also acrocentric and subtelocentric chromosomes.

Our present data confirm the occurrence of $2 n=52$ chromosomes for E. electricus. This chromosome number may be a prevalent feature in the family Gymnotidae. A phylogenetic analysis involving Gymnotus species (Fernandes-Matioli and Almeida-Toledo, 2001) has already been carried out, suggesting that the diploid number of 52 chromosomes may be a basal trait in this group. Further studies with other Gymnotidae species are necessary to test this hypothesis.

\section{Acknowledgments}

This research was supported by FAPESP $(05 / 55733$ 6), CNPq (300347/81-2), and CEPTA/IBAMA.

\section{References}

Albert JS and Campos-da-Paz R (1998) Systematics of Gymnotiformes with diagnoses of 58 clades: A review of available data. In: Malabarba LR, Reis RE, Vari RP, Lucena ZMS and Lucena CA (eds) Phylogeny and Classification of Neotropical Fishes. EDIPUCRS, Porto Alegre, pp 419-446.
Albert JS (2001) Species diversity and phylogenetic systematics of American knifefishes (Gymnotiformes, Teleostei). Misc Publ Mus Zool Univ Mich 190:1-127.

Almeida-Toledo LF, Daniel-Silva MFZ, Moysés CB, Fonteles SBA, Lopes CE, Akama A and Foresti F (2002) Chromosome evolution in fish: Sex chromosome variability in Eigenmannia virescens (Gymnotiformes, Sternopygidae). Cytogenet Genome Res 99:164-169.

Almeida-Toledo LF, Daniel-Silva MFZ, Moysés CB and Foresti F (2007) Chromosome variability in Gymnotiformes (Teleostei, Ostariophysi). In: Pisano E, Ozouf-Costaz C, Foresti F and Kapoor BG (eds) Fish Cytogenetics. Science Publishers, Enfield, pp 16-39.

Almeida-Toledo LF, Foresti F and Toledo-Filho SA (1981) Constitutive heterochromatin and nucleolus organizer regions in the knifefish, Apteronotus albifrons. Experientia 37:953-954.

Alves-Gomes JA, Ortí G, Haygood M, Meyer A and Heiligenberg W (1995) Phylogenetic analysis of the South American electric fishes (order Gymnotiformes) and the evolution of their electrogenic system. A synthesis based on morphology, electrophysiology, and mitochondrial sequence data. Mol Biol Evol 12:298-318.

Berra T (2001) Freshwater Fish Distribution. Academic Press, San Diego, 604 pp.

Brown ME (1957) The Physiology of Fishes. Academic Press, New York, 447 pp.

Campos da Paz R (2003) Family Gymnotidae. In: Reis RE, Kullander SO and Ferraris-Jr CJ (eds) Check list of the Freshwater Fishes of South and Central America. EDIPUCRS, Porto Alegre, pp 483-486.

Fernandes-Matioli FMC and Almeida-Toledo LF (2001) A molecular phylogeny analysis in Gymnotus species (Pisces, Gymnotiformes) with inferences on chromosome evolution. Caryologia 54:23-30.

Fernandes-Matioli FMC, Marcheto MCN, Almeida-Toledo LF and Toledo-Filho SA (1998) High intraspecific karyological conservation in four species of Gymnotus (Pisces, Gymnotiformes) from Southeastern Brazilian basins. Caryologia 51:221-234.

Foresti F, Almeida-Toledo LF and Toledo-Filho SA (1984) Chromosome studies in Gymnotus carapo and Gymnotus sp. (Pisces, Gymnotidae). Caryologia 37:141-146.

Foresti F, Oliveira C and Almeida-Toledo LF (1993) A method for chromosome preparations from large fish specimens using in vitro short-term treatment with colchicine. Experientia 49:810-813.

Howell WM and Black DA (1980) Controlled silver staining of nucleolus organizer regions with a protective colloidal developer: A 1-step method. Experientia 36:1014-1015.

Keynes R (1957) Electric organs. In: Brown ME (ed) The Physiology of Fishes. Academic Press, New York, pp 323-343.

Moller P (1995) Electric Fishes: History and Behavior. Chapman \& Hall, New York, 584 pp.

Sumner AT (1972) A simple technique demonstrating centromeric heterochromatin. Exp Cell Res 75:304-306.

Val AM and Almeida-Val VM (1995) Fishes of the Amazon and their Environment: Physiological and Biochemical Aspects. Springer, New York, 223 pp.

Associate Editor: Luiz Antonio Carlos Bertollo

License information: This is an open-access article distributed under the terms of the Creative Commons Attribution License, which permits unrestricted use, distribution, and reproduction in any medium, provided the original work is properly cited. 